\title{
Soft drinks: time trends and correlates in twenty-four European countries. A cross-national study using the DAFNE (Data Food Networking) databank
}

\author{
Androniki Naska ${ }^{1}$, Vasiliki Bountziouka ${ }^{1,2}$, Antonia Trichopoulou ${ }^{1,2, *}$ and the DAFNE \\ Participants $†$ \\ 'Department of Hygiene Epidemiology and Medical Statistics, School of Medicine, University of Athens, \\ 75 Mikras Asias Street, Athens 115 27, Greece: ${ }^{2}$ Hellenic Health Foundation, Athens, Greece
}

Submitted 22 September 2009: Accepted 17 February 2010: First published online 31 March 2010

\begin{abstract}
Objective: To evaluate time trends in the availability of soft drinks, to identify food choices associated with their consumption and to assess the relationship between socio-economic status and daily soft drink availability in a wide range of European countries.

Design: Data on food and beverage availability collected through the national household budget surveys and harmonized in the DAFNE (Data Food Networking) project were used. Averages and variability of soft drink availability were estimated and tests for time trends were performed. The daily availability of food groups which appear to be correlated with that of soft drinks was further estimated. Multivariate logistic and linear regression models were applied to evaluate the association between socio-economic status and the acquisition of soft drinks. Setting: Twenty-four European countries.

Subjects: Nationally representative samples of households.

Results: The availability of soft drinks is steadily and significantly increasing. Households in West and North Europe reported higher daily availability of soft drinks in comparison to other European regions. Soft drinks were also found to be correlated with lower availability of plant foods and milk and higher availability of meat and sugar products. Lower socio-economic status was associated with more frequent and higher availability of soft drinks in the household. Conclusions: Data collected in national samples of twenty-four European countries showed disparities in soft drink availability among socio-economic strata and European regions. The correlation of soft drinks with unfavourable dietary choices has public health implications, particularly among children and adolescents.
\end{abstract}

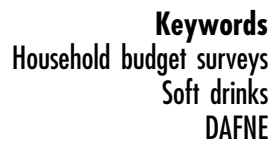

In several studies the consumption of soft drinks, particularly sugar-sweetened ones, has been positively associated with the risk of: weight gain and type 2 diabetes $^{(1-4)}$; osteoporosis ${ }^{(5)}$; CHD in women $^{(6)}$; dental caries and potential enamel erosion ${ }^{(7)}$; and gout in men ${ }^{(8)}$. Among these, the association between high consumption of soft drinks and weight gain is more frequently evaluated, since overweight and obesity are recognized as important public health challenges worldwide. In Europe, overweight affects 30-80\% of adults and about $20 \%$ of children and adolescents and the trend is particularly alarming since the current annual rate of increase is much higher than that in the $1970 \mathrm{~s}^{(9)}$. Several studies, mostly undertaken in the USA, indicate that the increase in the prevalence of overweight and obesity is

$\uparrow$ See Appendix for full list of DAFNE Participants. concurrent to an increase in the intake of added sugars. An important source of readily absorbable sugars in the daily diet is non-diet soft drinks, encompassing carbonated beverages, lemonades, orangeades, iced tea and similar drinks ${ }^{(2,7)}$.

In Europe, publications on the consumption of soft drinks make use of data collected through studies undertaken in specific countries, using various methodologies and mainly addressing children and adolescents $^{(10-12)}$. Few attempts have been made to compare the consumption of soft drinks among various European countries $^{(13,14)}$. The diet-related data regularly collected through national household budget surveys (HBS) offer a unique source of dietary information, allowing comparisons across essentially all European countries and several survey years. The use of the national HBS for nutrition monitoring purposes has been evaluated in the context of 
the EU-supported Data Food Networking (DAFNE) initiative ${ }^{(15)}$.

In the present analysis, DAFNE data from twenty-four European countries have been used to: (i) undertake inter-country comparisons and evaluate time trends in the availability of soft drinks; (ii) identify food choices associated with soft drinks consumption; and (iii) assess the relationship between socio-economic status and daily soft drink availability.

\section{Methods}

The DAFNE databank comprises data collected through standardized HBS, which are periodically conducted by the National Statistical Offices using country-representative population samples. HBS collect data on all household expenditures in order to calculate the consumer price index and analyse topics of social and economic interest $^{(16)}$. All HBS are confined to the population residing in private households, excluding collective or institutional ones. The sample is either obtained from registers of households or individuals with characteristics useful for stratification and sample selection, or through lists of households or dwellings in area units selected from the population census. Multistage stratified probability sampling is used in the large majority of surveys, with the household itself forming the ultimate sampling unit. In Germany and the Slovak Republic, a sampling scheme based on quota sampling is used instead. During data collection, the members of the participating households are asked to record, mainly in open questionnaires, all food and beverage purchases, contributions from the household's own production and items offered to members as gifts. Data on food quantities consumed when eating out (at restaurants, canteens and similar establishments) are, however, not collected. At present, within the European Union, the recording period for food and beverage acquisitions is in most countries $14 \mathrm{~d}$ and data collection is carried out throughout the year with due attention to capture seasonal variation in intake. In Belgium, Germany and Sweden the recording period is 1 month. Trained interviewers visit the households regularly to ensure complete data recording and to collect further information on demographic, socio-economic and lifestyle characteristics of the household members through standardized interviewer-administered questionnaires ${ }^{(16)}$.

The collected national data sets are centrally analysed according to the methodology developed and validated in the DAFNE project ${ }^{(15)}$. Briefly, data are read, cleaned and managed to a format suitable for a between-countries analysis. To improve comparability, food, demographic and socio-economic data are subsequently classified under common groupings with the application of criteria and iterative cross-coding allowing the formation of operational classification schemes. The data collected refer to quantities of foods and beverages available for consumption to the household members. The daily individual availability is calculated by dividing the household availability by the product of the referent time period and the mean household size, without making allowances for waste or food offered to domestic pets. The results thus estimated are stored in the DAFNE databank, which currently includes data sets of seventy-one surveys from twenty-four European countries.

The mean individual daily availability (in $\mathrm{ml}$ ) of soft drinks was estimated for all countries and most recent survey years, under the assumption that these are more relevant for contemporary patterns. Soft drinks were defined to include non-alcoholic carbonated, noncarbonated, sugar-sweetened and of low or no energy content beverages, energy drinks, squashes and syrups for the preparation of beverages, but excluding mineral water, fruit and vegetables juices. To assess the variation in dietary choices among households of different levels of soft drink acquisition, the participating households were classified in tertiles according to their daily per person soft drink availability. The mean availability of food groups which appeared to be correlated with household availability of soft drinks was estimated for households belonging to the first tertile. These mean values were used to calculate the proportional deviation (\%) of the corresponding mean availability in households of the second and third tertiles (higher soft drink consumers) from that of households in the first tertile (low soft drink consumers).

The households' socio-economic status was assessed through their food purchasing capacity, also referred to as food expenditure ratio ${ }^{(17,18)}$. It is expressed as the household's expenditure on food (including household acquisitions and expenses for eating out) divided by the total household expenses. The food expenditure ratio is a measure of households' financial welfare and has been used as an indicator of a household's socio-economic status or as a proxy for the household's income, with higher values suggesting lower socio-economic status or lower income ${ }^{(18-20)}$.

\section{Statistical analyses}

All analyses were conducted separately for each participating country and survey year with the Stata/SE $8 \cdot 0$ for Windows statistical software package (2003; Stata Corporation, College Station, TX, USA). Descriptive analyses included the estimation of averages and variability of soft drink availability (in $\mathrm{ml} /$ person per $\mathrm{d}$ ), by country and survey year. To assess trends over time, tests for trend were performed. To evaluate the association of socioeconomic status, as assessed through the households' food purchasing capacity, with the daily per person availability of soft drinks, data were initially modelled through multiple logistic regression contrasting availability to non-availability of soft drinks. Subsequently 
multiple linear regression models were applied among households reporting soft drink acquisitions, in order to evaluate the association of socio-economic status (in quintiles of the food expenditure ratio, categorically and as an ordered variable) with the logarithm of the daily availability of soft drinks (the distribution of which was positively skewed). Both logistic and linear regression models were performed separately for each country and survey year when information on the food expenditure ratio was available, also controlling for the trimester of participation (January-March, April-June, July-September and OctoberDecember, categorically).

\section{Results}

In Table 1, sample sizes, mean, median and the first and third quartiles of the daily individual availability of soft drinks are given for each of the twenty-four European countries and survey years. As expected, the distribution of soft drink availability in virtually all countries was positively skewed. Based on data collected in the late 1990 s or early 2000s, higher average availability of soft drinks at household level was generally recorded in Northern and Western European countries, in the Slovak Republic from Central/Eastern Europe, and in Malta from South Europe. In recent years, the Slovak Republic recorded the highest mean values of daily availability of soft drinks (227 ml/person per d, in 2003) and Latvia the lowest ( $28 \mathrm{ml} /$ person per $\mathrm{d}$, in 2004). With respect to time trends, in twelve out of the twenty-four participating countries the mean daily individual availability of soft drinks had increased and in most instances significantly so $(P<0.001$ in most instances); in five countries the mean availability decreased somewhat $(P \leq 0 \cdot 21)$ and in three countries the availability seems to have remained stable. In particular, comparisons of data collected at different time points showed that the household availability of soft drinks increased by $5 \%$ per year in Norway, Finland, the Republic of Ireland, Belgium, Italy and Greece, by about 8\% per year in Sweden, Latvia and the Slovak Republic, and by $23 \%$ per year in Portugal, although starting from a very low level.

Table 2 presents how the availability of certain foods varied according to increasing tertile of soft drink availability. The values in the table indicate the percentage deviations of the food group mean among households of the second and third tertile from the mean availability among households of the first, lower tertile. Results are presented for eleven food groups, common among countries. According to results presented in Table 2, as expected there appeared to be a pattern of a positive association of soft drinks with the indicated foods, reflecting a tendency of concomitant acquisition of larger or smaller food quantities. Nevertheless, stronger positive associations were generally evident with respect to processed meat, sugar and sugar products; that is, foods the excessive consumption of which is not considered desirable. Moreover, in several though not all countries, higher soft drink availability was associated with similar or lower availability of vegetables and fruits. Finally, in some countries there was an inverse association with milk availability. This could possibly indicate a displacement with soft drinks partially substituting milk, a finding with public health implications particularly among children and adolescents.

Table 3 shows the ratios of the odds of being a soft drink consumer $v$, the odds of not being one by quintile of household food expenditure ratio, with higher quintile indicating lower socio-economic status. Results refer to fourteen European countries with data on food expenditure ratio. Odds ratios above the null value of 1 indicate that the proportion of households consuming soft drinks is higher than in the reference category and vice versa. Table 4 shows the results of analysis undertaken in the same countries, but only among households reporting the acquisition of soft drinks during the survey period. Values in Table 4 indicate the percentage difference in the daily per person availability of soft drinks in comparison to the reference one. Both tables concordantly indicate that lower socio-economic status was associated with more frequent (Table 3, contrasting consumers $v$. non-consumers) and higher (Table 4, consumers only) availability of soft drinks in the household. An exception was noted, however, in Central and Eastern European countries during earlier HBS periods.

\section{Discussion}

High intake of sugar-sweetened soft drinks has been associated with weight gain and increased risk of type 2 diabetes ${ }^{(1-4)}$; increased risk of osteoporosis due to displacement of milk consumption and a subsequent lower calcium intake $^{(5,21-23)}$; higher risk of CHD in women ${ }^{(6)}$; increased risk of dental caries ${ }^{(7)}$; and increased risk of gout in men ${ }^{(8)}$. Using data collected in nationally representative samples of households in twenty-four European countries and at multiple time points, the availability of soft drinks at household level was found to be generally higher in Western and Northern as compared with Southern and Central/Eastern European regions. In most countries the mean daily availability of soft drinks has tended to increase in recent years. Higher soft drink availability was generally found to be positively associated with the daily availability of processed foods and negatively associated with fruits, vegetables and milk. When socio-economic differences in the availability of soft drinks were examined, households of lower socioeconomic status, reflected through the proportion of their expenses covering dietary needs, were associated with more frequent and higher availability of soft drinks in the household. With the exception of Central and Eastern 
Table 1 Mean and quartiles (P25, P50, P75) of the availability of soft drinks, by country and survey year (ml/person per d)

\begin{tabular}{|c|c|c|c|c|c|c|c|c|c|c|c|c|c|c|c|}
\hline \multirow[b]{2}{*}{ Country } & \multirow{2}{*}{$\begin{array}{l}\text { Year of } \\
\text { HBS }\end{array}$} & \multirow{2}{*}{$\begin{array}{c}\text { Number of participating } \\
\text { households }\end{array}$} & \multicolumn{4}{|c|}{ Soft drinks availability } & \multirow[b]{2}{*}{$P$ for trend ${ }^{*}$} & \multirow[b]{2}{*}{ Country } & \multirow[b]{2}{*}{ Year of HBS } & \multirow{2}{*}{$\begin{array}{c}\text { Number of participating } \\
\text { households }\end{array}$} & \multicolumn{4}{|c|}{ Soft drinks availability } & \multirow[b]{2}{*}{$P$ for trend ${ }^{*}$} \\
\hline & & & Mean & P25 & P50 & P75 & & & & & Mean & P25 & P50 & P75 & \\
\hline Austria & 1999 & 7098 & 116 & 0 & 36 & 151 & & Malta & 1994 & 2715 & 244 & 104 & 204 & 340 & $(-) 0.019$ \\
\hline \multirow[t]{3}{*}{ Belgium } & 1988 & 3235 & 129 & 41 & 91 & 170 & $(+)<0.001$ & & 1995 & 2748 & 239 & 101 & 200 & 331 & \\
\hline & 1997 & 2041 & 147 & 21 & 94 & 207 & & & 2000 & 2586 & 207 & 66 & 153 & 281 & \\
\hline & 1999 & 3745 & 191 & 31 & 129 & 266 & & Montenegro & 2003 & 380 & 29 & 8 & 20 & 37 & (0) 0.47 \\
\hline \multirow{2}{*}{ Croatia } & 1999 & 2937 & 125 & 0 & 72 & 198 & $(-) 0.037$ & & 2004 & 380 & 30 & 7 & 23 & 42 & \\
\hline & 2004 & 2847 & 116 & 0 & 67 & 178 & & Norway & 1987 & 4393 & 109 & 2 & 62 & 149 & $(+)<0.001$ \\
\hline \multirow{2}{*}{ Cyprus } & 1997 & 2645 & 113 & 15 & 88 & 154 & $(-)<0.001$ & & 1993 & 4033 & 152 & 27 & 107 & 214 & \\
\hline & 2003 & 2990 & 101 & 0 & 75 & 137 & & & 1997 & 3792 & 177 & 36 & 119 & 243 & \\
\hline \multirow[t]{3}{*}{ Finland } & 1985 & 8200 & 49 & 0 & 15 & 64 & $(+)<0.001$ & Poland & 1988 & 29645 & 34 & 1 & 16 & 43 & \\
\hline & 1990 & 8258 & 62 & 0 & 28 & 85 & & Portugal & 1990 & 12403 & 19 & 0 & 0 & 0 & $(+)<0.001$ \\
\hline & 1998 & 4359 & 81 & 0 & 36 & 107 & & & 1995 & 10554 & 47 & 0 & 0 & 47 & \\
\hline \multirow[t]{2}{*}{ France } & 1985 & 7288 & 31 & 0 & 0 & 0 & $(+)<0.001$ & & 2000 & 10020 & 63 & 0 & 12 & 89 & \\
\hline & 1991 & 6353 & 47 & 0 & 0 & 43 & & Serbia & 2003 & 3683 & 68 & 0 & 27 & 91 & $(-)<0.001$ \\
\hline \multirow[t]{3}{*}{ Germany } & $1988+$ & 17855 & 100 & 0 & 28 & 140 & (0) 0.73 & & 2004 & 4302 & 50 & 0 & 0 & 67 & \\
\hline & 1993 & 15825 & 124 & 0 & 38 & 175 & & Slovak Republic & 1997 & 1671 & 154 & 64 & 128 & 210 & $(+)<0.001$ \\
\hline & 1998 & 12680 & 108 & 0 & 22 & 144 & & & 2000 & 1647 & 207 & 96 & 169 & 269 & \\
\hline \multirow[t]{4}{*}{ Greece } & 1981 & 6034 & 31 & 0 & 0 & 28 & $(+) 0.13$ & & 2003 & 1645 & 227 & 99 & 183 & 309 & \\
\hline & 1987 & 6489 & 69 & 0 & 0 & 94 & & Slovenia & 1998 & 3867 & 91 & 0 & 14 & 98 & (0) 0.88 \\
\hline & 1998 & 6258 & 65 & 0 & 36 & 98 & & & 2000 & 3806 & 93 & 0 & 16 & 97 & \\
\hline & 2004 & 6555 & 66 & 0 & 36 & 99 & & & 2002 & 3687 & 90 & 0 & 8 & 91 & \\
\hline Hungary & 1991 & 11813 & 41 & 7 & 23 & 53 & & Spain & 1981 & 23972 & 93 & 0 & 24 & 142 & $(-) 0.21$ \\
\hline \multirow{3}{*}{ Ireland } & 1987 & 7705 & 70 & 18 & 50 & 95 & $(+)<0.001$ & & 1991 & 21155 & 84 & 0 & 7 & 114 & \\
\hline & 1994 & 7877 & 95 & 32 & 71 & 130 & & & 1999 & 14644 & 85 & 0 & 1 & 114 & \\
\hline & 1999 & 7644 & 122 & 43 & 92 & 168 & & Sweden & 1989 & 2079 & 77 & 13 & 51 & 102 & $(+)<0.001$ \\
\hline \multirow[t]{3}{*}{ Italy } & 1990 & 33172 & 32 & 0 & 0 & 7 & $(+)<0.001$ & & 1996 & 1104 & 115 & 15 & 80 & 159 & \\
\hline & 1993 & 34273 & 39 & 0 & 0 & 20 & & United Kingdom & 1992 & 7115 & 185 & 0 & 95 & 287 & $(+) 0.040$ \\
\hline & 1996 & 22740 & 42 & 0 & 0 & 33 & & & 1993 & 6925 & 189 & 0 & 95 & 286 & \\
\hline \multirow[t]{3}{*}{ Latvia } & 2002 & 3949 & 24 & 0 & 0 & 21 & $(+) 0.17$ & & 1994 & 7163 & 194 & 0 & 95 & 286 & \\
\hline & 2003 & 3631 & 28 & 0 & 0 & 29 & & & 1995 & 7320 & 212 & 0 & 107 & 321 & \\
\hline & 2004 & 3913 & 28 & 0 & 0 & 30 & & & 1996 & 7739 & 208 & 0 & 101 & 302 & \\
\hline \multirow{3}{*}{ Luxembourg } & 1993 & 3012 & 181 & 0 & 83 & 250 & & & 1997 & 7204 & 211 & 0 & 95 & 317 & \\
\hline & & & & & & & & & 1998 & 7059 & 200 & 0 & 95 & 286 & \\
\hline & & & & & & & & & 1999 & 7556 & 202 & 0 & 90 & 286 & \\
\hline
\end{tabular}

Source: The DAFNE databank (http://www.nut.uoa.gr/dafnesoft).

HBS, household budget survey.

*Trend analysis refers to the mean value: $(+)$, increasing availability; $(-)$, decreasing availability; $(0)$, no trend $(P>0 \cdot 30)$

tData collected in 1988 refer to the former West Germany. 
Table 2 Differences (\%) in the daily individual availability of the indicated food groups, by increasing tertile of soft drink availability. The mean availability of the indicated food groups among households of the first tertile was considered as referent

\begin{tabular}{|c|c|c|c|c|c|c|c|c|c|c|c|c|c|}
\hline Country & $\begin{array}{c}\text { Year of } \\
\text { HBS }\end{array}$ & Tertiles & $\begin{array}{c}\text { Cereal and } \\
\text { bakery } \\
\text { products }\end{array}$ & $\begin{array}{c}\text { Meat } \\
\text { products }\end{array}$ & Red meat & Cheese & Milk & $\begin{array}{l}\text { Dairy } \\
\text { products }\end{array}$ & $\begin{array}{l}\text { Lipids of } \\
\text { animal } \\
\text { origin }\end{array}$ & $\begin{array}{l}\text { Lipids of } \\
\text { vegetable } \\
\text { origin }\end{array}$ & Vegetables & Fruit & $\begin{array}{l}\text { Sugar and } \\
\text { sugar } \\
\text { products }\end{array}$ \\
\hline \multirow[t]{3}{*}{ Austria } & 1999 & $1 \mathrm{st}$ & referent & referent & referent & referent & referent & referent & referent & referent & referent & referent & referent \\
\hline & & 2nd & -0.1 & $-9 \cdot 5$ & $-11 \cdot 0$ & $-12 \cdot 6$ & $-14 \cdot 6$ & $-2 \cdot 6$ & $-25 \cdot 9$ & $-16 \cdot 3$ & $-11 \cdot 1$ & $-18 \cdot 7$ & $-18 \cdot 5$ \\
\hline & & $3 r d$ & +26.5 & +30.5 & $+16 \cdot 9$ & +0.6 & -0.1 & $+21 \cdot 3$ & $-14 \cdot 3$ & $+20 \cdot 1$ & +0.6 & $-9 \cdot 5$ & $+15 \cdot 2$ \\
\hline \multirow[t]{3}{*}{ Belgium } & 1999 & $1 \mathrm{st}$ & referent & referent & referent & referent & referent & referent & referent & referent & referent & referent & referent \\
\hline & & 2nd & $-1 \cdot 7$ & $+0 \cdot 3$ & $-12 \cdot 1$ & $-7 \cdot 3$ & -1.9 & $+1 \cdot 7$ & $-30 \cdot 3$ & $-10 \cdot 0$ & $-15 \cdot 0$ & $-25 \cdot 1$ & $-6 \cdot 8$ \\
\hline & & $3 r d$ & $+8 \cdot 1$ & $+24 \cdot 4$ & $+6 \cdot 4$ & $-5 \cdot 2$ & $-6 \cdot 2$ & $+2 \cdot 3$ & $-18 \cdot 6$ & $-7 \cdot 8$ & $-9 \cdot 5$ & $-24 \cdot 8$ & $+8 \cdot 8$ \\
\hline \multirow[t]{2}{*}{ Croatia } & 2004 & $1 \mathrm{st}$ & referent & referent & referent & referent & referent & referent & referent & referent & referent & referent & referent \\
\hline & & $\begin{array}{l}\text { 2nd } \\
3 \text { rd }\end{array}$ & $\begin{array}{l}+17.9 \\
+55.2\end{array}$ & $\begin{array}{r}-3 \cdot 2 \\
+30 \cdot 3\end{array}$ & $\begin{array}{l}-14 \cdot 1 \\
+29 \cdot 4\end{array}$ & $\begin{array}{r}+1 \cdot 0 \\
+26 \cdot 2\end{array}$ & $\begin{array}{r}-12 \cdot 1 \\
+3 \cdot 4\end{array}$ & $\begin{array}{r}-1.9 \\
+25.7\end{array}$ & $\begin{array}{l}-44 \cdot 5 \\
-12 \cdot 3\end{array}$ & $\begin{array}{l}-17 \cdot 2 \\
+2 ? \cdot 4\end{array}$ & $\begin{array}{r}-14 \cdot 0 \\
4 \cdot 0\end{array}$ & $\begin{array}{l}-11.2 \\
+20.7\end{array}$ & $-12 \cdot 6$ \\
\hline \multirow[t]{3}{*}{ Cyprus } & 2003 & $1 \mathrm{st}$ & referent & referent & referent & referent & referent & referent & referent & referent & $\begin{array}{l}4 \cdot 0 \\
\text { referent }\end{array}$ & referent & $\begin{array}{l}+17 \cdot 6 \\
\text { referent }\end{array}$ \\
\hline & & 2nd & $+25 \cdot 8$ & $+22 \cdot 1$ & $+4 \cdot 7$ & +8.9 & 3.0 & $+29 \cdot 8$ & +13.9 & $-6 \cdot 0$ & $-7 \cdot 6$ & $-3 \cdot 2$ & $+2 \cdot 9$ \\
\hline & & 3rd & $+71 \cdot 0$ & $+81 \cdot 9$ & $+54 \cdot 1$ & $+41 \cdot 7$ & $+11 \cdot 5$ & $+71 \cdot 4$ & $+36 \cdot 3$ & +39.5 & $+24 \cdot 7$ & $+31 \cdot 9$ & $+41 \cdot 4$ \\
\hline \multirow[t]{2}{*}{ Finland } & 1998 & $\begin{array}{l}1 \mathrm{st} \\
2 \mathrm{nd}\end{array}$ & $\begin{array}{r}\text { referent } \\
-6.2\end{array}$ & $\begin{array}{c}\text { referent } \\
-5 \cdot 8\end{array}$ & $\begin{array}{c}\text { referent } \\
-1.9\end{array}$ & $\begin{array}{c}\text { referent } \\
-2 \cdot 3\end{array}$ & $\begin{array}{c}\text { referent } \\
+7 \cdot 4\end{array}$ & $\begin{array}{c}\text { referent } \\
-3.5\end{array}$ & $\begin{array}{c}\text { referent } \\
-23 \cdot 4\end{array}$ & $\begin{array}{c}\text { referent } \\
-9 \cdot 1\end{array}$ & $\begin{array}{c}\text { referent } \\
-13 \cdot 3\end{array}$ & $\begin{array}{c}\text { referent } \\
-10 \cdot 1\end{array}$ & $\begin{array}{c}\text { referent } \\
+1 \cdot 8\end{array}$ \\
\hline & & $3 r d$ & $+19 \cdot 6$ & $+28 \cdot 4$ & $+28 \cdot 1$ & $+16 \cdot 7$ & $+4 \cdot 1$ & $+5 \cdot 0$ & $-10 \cdot 0$ & $-3 \cdot 7$ & $+1 \cdot 2$ & -4.9 & $+15 \cdot 2$ \\
\hline \multirow[t]{2}{*}{ France } & 1991 & $\begin{array}{l}\text { 1st } \\
\text { 2nd }\end{array}$ & $\begin{array}{r}\text { referent } \\
-19 \cdot 4\end{array}$ & $\begin{array}{c}\text { referent } \\
-7 \cdot 6\end{array}$ & $\begin{array}{c}\text { referent } \\
-19.5\end{array}$ & $\begin{array}{c}\text { referent } \\
-6 \cdot 1\end{array}$ & $\begin{array}{c}\text { referent } \\
+11 \cdot 0\end{array}$ & $\begin{array}{c}\text { referent } \\
+15.6\end{array}$ & $\begin{array}{c}\text { referent } \\
-16.5\end{array}$ & $\begin{array}{l}\text { referent } \\
+19 \cdot 8\end{array}$ & $\begin{array}{c}\text { referent } \\
-16 \cdot 0\end{array}$ & $\begin{array}{c}\text { referent } \\
-28.5\end{array}$ & $\begin{array}{l}\text { referent } \\
+2.5\end{array}$ \\
\hline & & 3 rd & $+2 \cdot 8$ & $+22 \cdot 1$ & $+10 \cdot 9$ & $+12 \cdot 8$ & $+15 \cdot 5$ & $+34 \cdot 2$ & $+4 \cdot 2$ & $+14 \cdot 3$ & +0.6 & $+6 \cdot 7$ & $+27 \cdot 4$ \\
\hline \multirow[t]{2}{*}{ Germany } & 1998 & $\begin{array}{l}\text { 1st } \\
\text { 2nd }\end{array}$ & $\begin{array}{r}\text { referent } \\
+3.3\end{array}$ & $\begin{array}{c}\text { referent } \\
+0.7\end{array}$ & $\begin{array}{c}\text { referent } \\
-4 \cdot 7\end{array}$ & $\begin{array}{c}\text { referent } \\
-10 \cdot 3\end{array}$ & $\begin{array}{c}\text { referent } \\
-9.5\end{array}$ & $\begin{array}{c}\text { referent } \\
+1 \cdot 4\end{array}$ & $\begin{array}{c}\text { referent } \\
-18.5\end{array}$ & $\begin{array}{c}\text { referent } \\
-8.3\end{array}$ & $\begin{array}{c}\text { referent } \\
-14 \cdot 4\end{array}$ & $\begin{array}{c}\text { referent } \\
-25 \cdot 1\end{array}$ & $\begin{array}{c}\text { referent } \\
-6.4\end{array}$ \\
\hline & & $3 r d$ & $+11 \cdot 2$ & $+19 \cdot 1$ & $+23 \cdot 1$ & $-15 \cdot 1$ & $-9 \cdot 2$ & $+4 \cdot 2$ & -14.9 & -0.3 & $-16 \cdot 7$ & $-25 \cdot 1$ & -0.5 \\
\hline \multirow[t]{3}{*}{ Greece } & 2004 & $1 \mathrm{st}$ & referent & referent & referent & referent & referent & referent & referent & referent & referent & referent & referent \\
\hline & & 2nd & $+11 \cdot 2$ & $+33 \cdot 1$ & $-6 \cdot 1$ & $-1 \cdot 7$ & $+0 \cdot 1$ & -1.5 & $+15 \cdot 1$ & $-14 \cdot 2$ & $-12 \cdot 4$ & $-11 \cdot 1$ & $-6 \cdot 9$ \\
\hline & & 3 rd & $+43 \cdot 8$ & $+50 \cdot 3$ & $+24 \cdot 0$ & $+28 \cdot 5$ & $+5 \cdot 3$ & $+17 \cdot 8$ & $+11 \cdot 6$ & $+7 \cdot 8$ & $+6 \cdot 2$ & $+5 \cdot 3$ & $+19 \cdot 5$ \\
\hline \multirow[t]{2}{*}{ Hungary } & 1991 & $\begin{array}{l}\text { 1st } \\
\text { 2nd }\end{array}$ & $\begin{array}{r}\text { referent } \\
+21.9\end{array}$ & $\begin{array}{c}\text { referent } \\
+9 \cdot 3\end{array}$ & $\begin{array}{c}\text { referent } \\
+3.8\end{array}$ & $\begin{array}{c}\text { referent } \\
+13 \cdot 2\end{array}$ & $\begin{array}{c}\text { referent } \\
-4 \cdot 7\end{array}$ & $\begin{array}{l}\text { referent } \\
\quad+8.6\end{array}$ & $\begin{array}{c}\text { referent } \\
-5.5\end{array}$ & $\begin{array}{l}\text { referent } \\
+2 \cdot 2\end{array}$ & $\begin{array}{c}\text { referent } \\
+2 \cdot 1\end{array}$ & $\begin{array}{c}\text { referent } \\
+6.6\end{array}$ & $\begin{array}{l}\text { referent } \\
+2 \cdot 8\end{array}$ \\
\hline & & 3 rd & $+45 \cdot 0$ & +24.9 & $+24 \cdot 1$ & $+52 \cdot 2$ & $-1 \cdot 3$ & $+29 \cdot 3$ & $+3 \cdot 3$ & $+11 \cdot 6$ & $+20 \cdot 8$ & $+30 \cdot 8$ & $+15 \cdot 5$ \\
\hline \multirow[t]{3}{*}{ Ireland } & 1999 & $1 \mathrm{st}$ & referent & referent & referent & referent & referent & referent & referent & referent & referent & referent & referent \\
\hline & & 2nd & $+11 \cdot 1$ & $+0 \cdot 2$ & $0 \cdot 0$ & $-1 \cdot 4$ & $-3 \cdot 2$ & $+10 \cdot 6$ & $-19 \cdot 4$ & -0.4 & $-4 \cdot 7$ & $-5 \cdot 5$ & $+6 \cdot 7$ \\
\hline & & 3rd & $+35 \cdot 4$ & $+22 \cdot 1$ & $+9 \cdot 4$ & $+16 \cdot 6$ & $-1 \cdot 0$ & $+24 \cdot 0$ & $-4 \cdot 5$ & $+22 \cdot 5$ & +13.9 & $+5 \cdot 4$ & $+24 \cdot 4$ \\
\hline \multirow[t]{3}{*}{ Italy } & 1996 & 1st & referent & referent & referent & referent & referent & referent & referent & referent & referent & referent & referent \\
\hline & & 2nd & $+6 \cdot 3$ & $+10 \cdot 4$ & $-8 \cdot 3$ & $+5 \cdot 8$ & $-4 \cdot 2$ & $+43 \cdot 0$ & $-10 \cdot 1$ & $-22 \cdot 1$ & $-1 \cdot 8$ & $-7 \cdot 0$ & $-14 \cdot 4$ \\
\hline & & 3rd & $+14 \cdot 4$ & $+27 \cdot 8$ & $+5 \cdot 6$ & $+17 \cdot 6$ & -0.5 & $+57 \cdot 8$ & $+3 \cdot 4$ & $-3 \cdot 9$ & +8.4 & $+5 \cdot 6$ & $+3 \cdot 0$ \\
\hline \multirow[t]{3}{*}{ Latvia } & 2004 & 1st & referent & referent & referent & referent & referent & referent & referent & referent & referent & referent & referent \\
\hline & & 2nd & $+23 \cdot 4$ & $-7 \cdot 1$ & $-16 \cdot 4$ & $-18 \cdot 3$ & $-21 \cdot 7$ & $-10 \cdot 4$ & $-32 \cdot 0$ & $-35 \cdot 8$ & $-27 \cdot 2$ & $-5 \cdot 0$ & $-31 \cdot 9$ \\
\hline & & 3rd & $+57 \cdot 1$ & $+22 \cdot 8$ & $+1 \cdot 9$ & $-2 \cdot 4$ & $-21 \cdot 4$ & $+8 \cdot 2$ & $-20 \cdot 9$ & $-24 \cdot 7$ & $-13 \cdot 4$ & $+20 \cdot 0$ & $-9 \cdot 9$ \\
\hline \multirow[t]{2}{*}{ Luxembourg } & 1993 & $\begin{array}{l}\text { 1st } \\
2 \text { nd }\end{array}$ & $\begin{array}{r}\text { referent } \\
-34.8\end{array}$ & $\begin{array}{l}\text { referent } \\
+15 \cdot 8\end{array}$ & $\begin{array}{c}\text { referent } \\
-7 \cdot 2\end{array}$ & $\begin{array}{c}\text { referent } \\
-9 \cdot 1\end{array}$ & $\begin{array}{c}\text { referent } \\
+7.6\end{array}$ & $\begin{array}{c}\text { referent } \\
0.0\end{array}$ & $\begin{array}{c}\text { referent } \\
+8.2\end{array}$ & $\begin{array}{c}\text { referent } \\
-5.0\end{array}$ & $\begin{array}{c}\text { referent } \\
-28.4\end{array}$ & $\begin{array}{c}\text { referent } \\
-14.9\end{array}$ & $\begin{array}{c}\text { referent } \\
+15.1\end{array}$ \\
\hline & & $3 \mathrm{rd}$ & $-4 \cdot 2$ & +56.6 & $+17 \cdot 1$ & $+13 \cdot 1$ & +0.9 & +28.0 & $-14 \cdot 1$ & $+16 \cdot 2$ & $-19 \cdot 9$ & $+5 \cdot 0$ & $+19 \cdot 3$ \\
\hline \multirow[t]{3}{*}{ Malta } & 2000 & 1st & referent & referent & referent & referent & referent & referent & referent & referent & referent & referent & referent \\
\hline & & $\begin{array}{l}\text { 2nd } \\
\text { 3rd }\end{array}$ & $\begin{array}{r}+6.4 \\
+25.6\end{array}$ & $+19 \cdot 4$ & $+20 \cdot 2$ & $+6 \cdot 2$ & $\begin{array}{r}-19 \cdot 5 \\
-8.0\end{array}$ & $+13 \cdot 4$ & $+11 \cdot 9$ & $+20 \cdot 6$ & +0.2 & $+5 \cdot 3$ & $+11 \cdot 0$ \\
\hline & & 3rd & $+25 \cdot 6$ & $+66 \cdot 3$ & $+60 \cdot 1$ & $+30 \cdot 8$ & -8.0 & $+56 \cdot 2$ & $+39 \cdot 4$ & $+56 \cdot 5$ & $+32 \cdot 3$ & $+51 \cdot 4$ & $+44 \cdot 4$ \\
\hline \multirow[t]{3}{*}{ Montenegro } & 2004 & 1st & referent & referent & referent & referent & referent & referent & referent & referent & referent & referent & referent \\
\hline & & 2nd & $\begin{array}{l}+187 \cdot 8 \\
+164 \cdot 3\end{array}$ & $+28 \cdot 1$ & $+21 \cdot 2$ & $+28 \cdot 3$ & -3.0 & $-15 \cdot 6$ & $-42 \cdot 6$ & $+66 \cdot 4$ & $+25 \cdot 1$ & $+43 \cdot 8$ & $+55 \cdot 4$ \\
\hline & & 3rd & $+164 \cdot 3$ & $+2 \cdot 3$ & $+30 \cdot 8$ & $-17 \cdot 2$ & $-22 \cdot 1$ & $+50 \cdot 1$ & $-29 \cdot 5$ & +28.2 & $-1 \cdot 4$ & $-7 \cdot 1$ & +14.5 \\
\hline
\end{tabular}


Table 2 Continued

\begin{tabular}{|c|c|c|c|c|c|c|c|c|c|c|c|c|c|}
\hline Country & $\begin{array}{c}\text { Year of } \\
\text { HBS }\end{array}$ & Tertiles & $\begin{array}{c}\text { Cereal and } \\
\text { bakery } \\
\text { products }\end{array}$ & $\begin{array}{c}\text { Meat } \\
\text { products }\end{array}$ & Red meat & Cheese & Milk & $\begin{array}{c}\text { Dairy } \\
\text { products }\end{array}$ & $\begin{array}{l}\text { Lipids of } \\
\text { animal } \\
\text { origin }\end{array}$ & $\begin{array}{l}\text { Lipids of } \\
\text { vegetable } \\
\text { origin }\end{array}$ & Vegetables & Fruit & $\begin{array}{l}\text { Sugar and } \\
\text { sugar } \\
\text { products }\end{array}$ \\
\hline Norway & 1997 & $\begin{array}{l}\text { 1st } \\
\text { 2nd } \\
\text { 3rd }\end{array}$ & $\begin{array}{r}\text { referent } \\
-1.3 \\
+20.6\end{array}$ & $\begin{array}{c}\text { referent } \\
+3.5 \\
+39.2\end{array}$ & $\begin{array}{c}\text { referent } \\
-2 \cdot 1 \\
+13.0\end{array}$ & $\begin{array}{c}\text { referent } \\
-4 \cdot 1 \\
+4 \cdot 6\end{array}$ & $\begin{array}{c}\text { referent } \\
+3 \cdot 0 \\
+9 \cdot 1\end{array}$ & $\begin{array}{c}\text { referent } \\
-6 \cdot 3 \\
+10 \cdot 8\end{array}$ & $\begin{array}{c}\text { referent } \\
-18.7 \\
-1.9\end{array}$ & $\begin{array}{c}\text { referent } \\
-5.3 \\
+5.5\end{array}$ & $\begin{array}{c}\text { referent } \\
-14 \cdot 0 \\
-2 \cdot 6\end{array}$ & $\begin{array}{c}\text { referent } \\
-6.6 \\
-2.6\end{array}$ & $\begin{array}{l}\text { referent } \\
+12.0 \\
+20.5\end{array}$ \\
\hline Poland & 1988 & $\begin{array}{l}\text { 1st } \\
\text { 2nd } \\
\text { 3rd }\end{array}$ & $\begin{array}{r}\text { referent } \\
+11.6 \\
+49.9\end{array}$ & $\begin{array}{c}\text { referent } \\
-1.5 \\
+11.7\end{array}$ & $\begin{array}{c}\text { referent } \\
-5 \cdot 4 \\
+6 \cdot 7\end{array}$ & $\begin{array}{c}\text { referent } \\
-12 \cdot 0 \\
-0.6\end{array}$ & $\begin{array}{c}\text { referent } \\
-9 \cdot 1 \\
-10 \cdot 6\end{array}$ & $\begin{array}{c}\text { referent } \\
-6 \cdot 2 \\
+5 \cdot 8\end{array}$ & $\begin{array}{c}\text { referent } \\
-9.9 \\
-4.8\end{array}$ & $\begin{array}{c}\text { referent } \\
-10.1 \\
-0.9\end{array}$ & $\begin{array}{c}\text { referent } \\
-6.9 \\
+6.5\end{array}$ & $\begin{array}{c}\text { referent } \\
-1.4 \\
+16.9\end{array}$ & $\begin{array}{c}\text { referent } \\
-6 \cdot 8 \\
+2 \cdot 8\end{array}$ \\
\hline Portugal & 2000 & $\begin{array}{l}\text { 1st } \\
\text { 2nd } \\
\text { 3rd }\end{array}$ & $\begin{array}{r}\text { referent } \\
+18.6 \\
+63.6\end{array}$ & $\begin{array}{c}\text { referent } \\
+15 \cdot 4 \\
+65 \cdot 8\end{array}$ & $\begin{array}{c}\text { referent } \\
-11.5 \\
+24.0\end{array}$ & $\begin{array}{c}\text { referent } \\
-21.9 \\
+2.9\end{array}$ & $\begin{array}{c}\text { referent } \\
-13 \cdot 1 \\
+3.9\end{array}$ & $\begin{array}{l}\text { referent } \\
+22 \cdot 2 \\
+65 \cdot 2\end{array}$ & $\begin{array}{c}\text { referent } \\
+4 \cdot 1 \\
+44 \cdot 0\end{array}$ & $\begin{array}{c}\text { referent } \\
-11.6 \\
+19.7\end{array}$ & $\begin{array}{c}\text { referent } \\
-17 \cdot 2 \\
+3 \cdot 8\end{array}$ & $\begin{array}{c}\text { referent } \\
-20.7 \\
-0.8\end{array}$ & $\begin{array}{c}\text { referent } \\
-14.5 \\
+12.9\end{array}$ \\
\hline Serbia & 2004 & $\begin{array}{l}\text { 1st } \\
\text { 2nd } \\
\text { 3rd }\end{array}$ & $\begin{array}{r}\text { referent } \\
+5.5 \\
+58.8\end{array}$ & $\begin{array}{c}\text { referent } \\
-18 \cdot 5 \\
+13 \cdot 2\end{array}$ & $\begin{array}{l}\text { referent } \\
-20 \cdot 2 \\
+50 \cdot 6\end{array}$ & $\begin{array}{l}\text { referent } \\
-13 \cdot 2 \\
+12 \cdot 0\end{array}$ & $\begin{array}{c}\text { referent } \\
-26 \cdot 6 \\
-11 \cdot 1\end{array}$ & $\begin{array}{c}\text { referent } \\
-6.7 \\
+2.6\end{array}$ & $\begin{array}{c}\text { referent } \\
-14 \cdot 7 \\
+44 \cdot 6\end{array}$ & $\begin{array}{c}\text { referent } \\
-15.5 \\
-0.4\end{array}$ & $\begin{array}{l}\text { referent } \\
-14 \cdot 2 \\
+18 \cdot 3\end{array}$ & $\begin{array}{c}\text { referent } \\
-10.0 \\
+32.7\end{array}$ & $\begin{array}{l}\text { referent } \\
+16.6 \\
+48.4\end{array}$ \\
\hline Slovak Republic & 2003 & $\begin{array}{l}1 \mathrm{st} \\
\text { 2nd } \\
\text { 3rd }\end{array}$ & $\begin{array}{r}\text { referent } \\
+12 \cdot 3 \\
+27 \cdot 2\end{array}$ & $\begin{array}{c}\text { referent } \\
+5.0 \\
+25.2\end{array}$ & $\begin{array}{c}\text { referent } \\
+4 \cdot 7 \\
+17 \cdot 4\end{array}$ & $\begin{array}{c}\text { referent } \\
-2 \cdot 8 \\
+7 \cdot 8\end{array}$ & $\begin{array}{c}\text { referent } \\
-8.3 \\
-4.5\end{array}$ & $\begin{array}{c}\text { referent } \\
+1 \cdot 7 \\
+12 \cdot 9\end{array}$ & $\begin{array}{c}\text { referent } \\
\quad-0.9 \\
+1.0\end{array}$ & $\begin{array}{c}\text { referent } \\
-0.7 \\
+17 \cdot 7\end{array}$ & $\begin{array}{c}\text { referent } \\
-6 \cdot 1 \\
-1 \cdot 3\end{array}$ & $\begin{array}{c}\text { referent } \\
-4 \cdot 0 \\
+2 \cdot 4\end{array}$ & $\begin{array}{l}\text { referent } \\
+4.0 \\
+19.4\end{array}$ \\
\hline Slovenia & 2002 & $\begin{array}{l}\text { 1st } \\
\text { 2nd } \\
\text { 3rd }\end{array}$ & $\begin{array}{r}\text { referent } \\
-24.5 \\
+31.9\end{array}$ & $\begin{array}{c}\text { referent } \\
-35.9 \\
+15.8\end{array}$ & $\begin{array}{c}\text { referent } \\
-39 \cdot 2 \\
-29 \cdot 4\end{array}$ & $\begin{array}{c}\text { referent } \\
-40 \cdot 6 \\
+17 \cdot 2\end{array}$ & $\begin{array}{c}\text { referent } \\
-44 \cdot 7 \\
-10 \cdot 3\end{array}$ & $\begin{array}{c}\text { referent } \\
-29.7 \\
+15.9\end{array}$ & $\begin{array}{c}\text { referent } \\
-43 \cdot 1 \\
-32 \cdot 1\end{array}$ & $\begin{array}{c}\text { referent } \\
-47.5 \\
+6.2\end{array}$ & $\begin{array}{c}\text { referent } \\
-36 \cdot 7 \\
+1 \cdot 4\end{array}$ & $\begin{array}{c}\text { referent } \\
-40.6 \\
-0.3\end{array}$ & $\begin{array}{c}\text { referent } \\
-44.2 \\
-3.5\end{array}$ \\
\hline Spain & 1999 & $\begin{array}{l}\text { 1st } \\
\text { 2nd } \\
\text { 3rd }\end{array}$ & $\begin{array}{r}\text { referent } \\
-14 \cdot 4 \\
+39 \cdot 4\end{array}$ & $\begin{array}{c}\text { referent } \\
-17 \cdot 8 \\
+30 \cdot 9\end{array}$ & $\begin{array}{c}\text { referent } \\
-17 \cdot 8 \\
+12 \cdot 5\end{array}$ & $\begin{array}{c}\text { referent } \\
-22 \cdot 7 \\
+24 \cdot 3\end{array}$ & $\begin{array}{c}\text { referent } \\
-15 \cdot 6 \\
+18 \cdot 8\end{array}$ & $\begin{array}{c}\text { referent } \\
-2 \cdot 2 \\
+57 \cdot 3\end{array}$ & $\begin{array}{c}\text { referent } \\
-55.0 \\
-25.6\end{array}$ & $\begin{array}{c}\text { referent } \\
-16.3 \\
+35.9\end{array}$ & $\begin{array}{l}\text { referent } \\
-26.0 \\
+1.7\end{array}$ & $\begin{array}{c}\text { referent } \\
-23.3 \\
-0.1\end{array}$ & $\begin{array}{c}\text { referent } \\
-7.7 \\
+56.9\end{array}$ \\
\hline Sweden & 1996 & $\begin{array}{l}\text { 1st } \\
\text { 2nd } \\
\text { 3rd }\end{array}$ & $\begin{array}{r}\text { referent } \\
+8 \cdot 1 \\
+31 \cdot 0\end{array}$ & $\begin{array}{c}\text { referent } \\
+6 \cdot 0 \\
+34.6\end{array}$ & $\begin{array}{c}\text { referent } \\
-2 \cdot 6 \\
+10 \cdot 8\end{array}$ & $\begin{array}{c}\text { referent } \\
-6.9 \\
+4.3\end{array}$ & $\begin{array}{c}\text { referent } \\
+18 \cdot 7 \\
+12 \cdot 4\end{array}$ & $\begin{array}{l}\text { referent } \\
\quad+6 \cdot 8 \\
+9 \cdot 8\end{array}$ & $\begin{array}{c}\text { referent } \\
-14 \cdot 0 \\
-14.9\end{array}$ & $\begin{array}{c}\text { referent } \\
-3.9 \\
+18.7\end{array}$ & $\begin{array}{c}\text { referent } \\
-0.3 \\
+20.2\end{array}$ & $\begin{array}{c}\text { referent } \\
\quad+6 \cdot 4 \\
+9.5\end{array}$ & $\begin{array}{c}\text { referent } \\
-5.6 \\
+20.5\end{array}$ \\
\hline United Kingdom & 1999 & $\begin{array}{l}1 \text { st } \\
\text { 2nd } \\
\text { 3rd }\end{array}$ & $\begin{array}{l}\text { referent } \\
+17 \cdot 0 \\
+41 \cdot 3\end{array}$ & $\begin{array}{c}\text { referent } \\
+1 \cdot 3 \\
+28 \cdot 2\end{array}$ & $\begin{array}{c}\text { referent } \\
+4 \cdot 3 \\
+26 \cdot 6\end{array}$ & $\begin{array}{c}\text { referent } \\
+4 \cdot 0 \\
+31 \cdot 8\end{array}$ & $\begin{array}{c}\text { referent } \\
-0.7 \\
+5.0\end{array}$ & $\begin{array}{c}\text { referent } \\
+20 \cdot 9 \\
+51 \cdot 1\end{array}$ & $\begin{array}{c}\text { referent } \\
+2 \cdot 5 \\
+17 \cdot 2\end{array}$ & $\begin{array}{l}\text { referent } \\
+24 \cdot 8 \\
+37 \cdot 8\end{array}$ & $\begin{array}{c}\text { referent } \\
-4.0 \\
+14.9\end{array}$ & $\begin{array}{c}\text { referent } \\
-2 \cdot 4 \\
+12 \cdot 4\end{array}$ & $\begin{array}{c}\text { referent } \\
+15.4 \\
+33.9\end{array}$ \\
\hline
\end{tabular}

Source: The DAFNE databank (http://www.nut.uoa.gr/dafnesoft). 
Table 3 Odds ratios and $95 \%$ confidence intervals contrasting households reporting $v$. not reporting the acquisition of soft drinks, by quintile of the household food expenditure ratio*, using the first quintile as referent

\begin{tabular}{|c|c|c|c|c|c|c|c|c|c|c|c|}
\hline \multirow[b]{2}{*}{ Country } & \multirow[b]{2}{*}{ Year of HBS } & \multicolumn{2}{|c|}{ 2nd quintile } & \multicolumn{2}{|c|}{ 3rd quintile } & \multicolumn{2}{|c|}{ 4th quintile } & \multicolumn{2}{|c|}{ 5th quintile } & \multicolumn{2}{|c|}{ Ordered quintiles } \\
\hline & & OR & $95 \% \mathrm{Cl}$ & OR & $95 \% \mathrm{Cl}$ & OR & $95 \% \mathrm{Cl}$ & OR & $95 \% \mathrm{Cl}$ & OR & $95 \% \mathrm{Cl}$ \\
\hline Austria & 1999 & 1.09 & $0.91,1.31$ & $1 \cdot 54$ & $1 \cdot 30,1 \cdot 84$ & 1.65 & $1.38,1.97$ & $1 \cdot 41$ & $1 \cdot 18,1 \cdot 69$ & $1 \cdot 11$ & $1 \cdot 07,1 \cdot 16$ \\
\hline \multirow[t]{2}{*}{ Cyprus } & 1997 & $1 \cdot 59$ & $1 \cdot 38,1 \cdot 85$ & $1 \cdot 61$ & $1 \cdot 39,1 \cdot 87$ & $1 \cdot 85$ & $1 \cdot 58,2 \cdot 17$ & 1.50 & $1 \cdot 27,1 \cdot 77$ & $1 \cdot 12$ & $1.08,1.17$ \\
\hline & 2003 & 1.59 & $1 \cdot 38,1 \cdot 82$ & 1.54 & $1 \cdot 34,1 \cdot 78$ & $2 \cdot 00$ & $1 \cdot 73,2 \cdot 32$ & $1 \cdot 64$ & $1 \cdot 40,1.91$ & $1 \cdot 15$ & $1 \cdot 11,1 \cdot 19$ \\
\hline \multirow{3}{*}{ Finland } & 1985 & $1 \cdot 50$ & $1 \cdot 36,1 \cdot 66$ & $1 \cdot 52$ & $1 \cdot 37,1 \cdot 68$ & 1.51 & $1.36,1.67$ & 1.29 & $1 \cdot 16,1 \cdot 43$ & 1.05 & $1.03,1.08$ \\
\hline & 1990 & $1 \cdot 64$ & $1 \cdot 48,1 \cdot 82$ & $2 \cdot 11$ & $1 \cdot 90,2 \cdot 34$ & $2 \cdot 18$ & $1 \cdot 97,2 \cdot 42$ & 1.92 & $1 \cdot 73,2 \cdot 14$ & $1 \cdot 18$ & $1 \cdot 15,1 \cdot 21$ \\
\hline & 1998 & $1 \cdot 79$ & $1.55,2.07$ & $1 \cdot 75$ & $1.51,2.01$ & $2 \cdot 21$ & $1.91,2.56$ & $2 \cdot 19$ & $1 \cdot 88,2 \cdot 54$ & $1 \cdot 20$ & $1 \cdot 16,1.24$ \\
\hline Germany & 1998 & $1 \cdot 22$ & $1 \cdot 12,1 \cdot 34$ & $1 \cdot 42$ & $1 \cdot 30,1 \cdot 55$ & $1 \cdot 72$ & $1 \cdot 58,1 \cdot 88$ & $2 \cdot 07$ & $1 \cdot 89,2 \cdot 27$ & $1 \cdot 20$ & $1 \cdot 17,1 \cdot 22$ \\
\hline \multirow[t]{2}{*}{ Greece } & 1998 & $1 \cdot 10$ & $1 \cdot 00,1 \cdot 21$ & $1 \cdot 28$ & $1 \cdot 16,1 \cdot 41$ & $1 \cdot 45$ & $1 \cdot 31,1 \cdot 60$ & $1 \cdot 32$ & $1 \cdot 20,1 \cdot 46$ & 1.09 & $1 \cdot 06,1 \cdot 11$ \\
\hline & 2004 & $1 \cdot 19$ & $1 \cdot 07,1 \cdot 32$ & $1 \cdot 10$ & $1 \cdot 00,1 \cdot 22$ & $1 \cdot 21$ & $1 \cdot 09,1 \cdot 34$ & 1.25 & $1 \cdot 12,1 \cdot 38$ & $1 \cdot 05$ & $1.02,1.07$ \\
\hline \multirow[t]{2}{*}{ Ireland } & 1994 & 1.91 & $1 \cdot 70,2 \cdot 16$ & $2 \cdot 27$ & $2 \cdot 01,2 \cdot 56$ & $2 \cdot 13$ & $1 \cdot 89,2 \cdot 40$ & 1.47 & $1 \cdot 31,1 \cdot 65$ & 1.09 & $1 \cdot 06,1 \cdot 13$ \\
\hline & 1999 & $2 \cdot 19$ & $1.91,2.50$ & $2 \cdot 07$ & $1 \cdot 81,2 \cdot 36$ & $2 \cdot 28$ & $1.98,2.62$ & 0.97 & $0.86,1 \cdot 10$ & $1 \cdot 01$ & $0.97,1.05$ \\
\hline \multirow[t]{3}{*}{ Italy } & 1990 & 1.04 & $0 \cdot 98,1 \cdot 10$ & 1.05 & $0.99,1 \cdot 11$ & 1.03 & $0.98,1.09$ & $1 \cdot 00$ & $0.94,1.05$ & $1 \cdot 00$ & $0.99,1.01$ \\
\hline & 1993 & 1.09 & $1 \cdot 04,1 \cdot 16$ & $1 \cdot 10$ & $1 \cdot 04,1 \cdot 16$ & $1 \cdot 19$ & $1 \cdot 12,1 \cdot 25$ & 1.04 & $0.98,1 \cdot 10$ & $1 \cdot 01$ & $1.00,1.03$ \\
\hline & 1996 & 1.03 & $0.96,1 \cdot 11$ & $1 \cdot 25$ & $1 \cdot 17,1 \cdot 35$ & $1 \cdot 21$ & $1 \cdot 13,1 \cdot 30$ & $1 \cdot 27$ & $1 \cdot 18,1 \cdot 36$ & $1 \cdot 07$ & $1.05,1.08$ \\
\hline \multirow[t]{3}{*}{ Malta } & 1994 & $1 \cdot 04$ & $0 \cdot 63,1 \cdot 70$ & $1 \cdot 37$ & $0 \cdot 86,2 \cdot 18$ & $1 \cdot 47$ & $0 \cdot 89,2 \cdot 42$ & $0 \cdot 87$ & $0.55,1.36$ & 1.00 & $0 \cdot 89,1 \cdot 12$ \\
\hline & 1995 & $1 \cdot 27$ & $0.82,1.97$ & 1.02 & $0.66,1.57$ & 1.97 & $1 \cdot 20,3 \cdot 24$ & 0.91 & $0.60,1.39$ & $1 \cdot 01$ & $0.91,1.13$ \\
\hline & 2000 & $1 \cdot 78$ & $1 \cdot 42,2 \cdot 22$ & $2 \cdot 78$ & $2 \cdot 19,2 \cdot 22$ & $3 \cdot 32$ & $2 \cdot 52,4 \cdot 38$ & $1 \cdot 81$ & $1 \cdot 43,2 \cdot 28$ & $1 \cdot 23$ & $1 \cdot 16,1 \cdot 32$ \\
\hline Norway & 1997 & 1.98 & $1 \cdot 61,2 \cdot 45$ & 1.92 & $1 \cdot 53,2 \cdot 41$ & $2 \cdot 58$ & $2 \cdot 01,3 \cdot 29$ & $1 \cdot 75$ & $1 \cdot 40,2 \cdot 19$ & $1 \cdot 17$ & $1 \cdot 10,1 \cdot 24$ \\
\hline \multirow[t]{3}{*}{ Portugal } & 1990 & $1 \cdot 19$ & $1 \cdot 08,1 \cdot 32$ & $1 \cdot 28$ & $1 \cdot 16,1 \cdot 41$ & $1 \cdot 23$ & $1 \cdot 12,1 \cdot 36$ & 1.03 & $0.93,1.14$ & $1 \cdot 01$ & $0.99,1.03$ \\
\hline & 1995 & $1 \cdot 01$ & $0.91,1.11$ & $1 \cdot 01$ & $0.91,1 \cdot 11$ & $1 \cdot 06$ & $0.96,1 \cdot 17$ & $0 \cdot 76$ & $0.68,0.84$ & 0.95 & $0.93,0.97$ \\
\hline & 2000 & $1 \cdot 13$ & $1 \cdot 01,1 \cdot 26$ & $1 \cdot 28$ & $1 \cdot 14,1 \cdot 42$ & $1 \cdot 25$ & $1 \cdot 12,1 \cdot 40$ & 1.29 & $1 \cdot 15,1 \cdot 45$ & $1 \cdot 06$ & $1.04,1.09$ \\
\hline \multirow[t]{3}{*}{ Slovak Republic } & 1997 & $1 \cdot 38$ & $0 \cdot 65,2 \cdot 91$ & $1 \cdot 83$ & $0 \cdot 80,4 \cdot 22$ & $4 \cdot 82$ & $1.59,14.59$ & 0.74 & $0.38,1.45$ & 0.99 & $0.80,1.23$ \\
\hline & 2000 & $2 \cdot 22$ & $0 \cdot 48,10 \cdot 21$ & $1 \cdot 14$ & $0 \cdot 31,4 \cdot 16$ & $5 \cdot 00$ & $1 \cdot 68,14 \cdot 82$ & 0.41 & $0.18,0.92$ & $0 \cdot 76$ & $0.57,1.01$ \\
\hline & 2003 & 1.09 & $0 \cdot 43,2 \cdot 74$ & $2 \cdot 19$ & $0 \cdot 28,17 \cdot 27$ & 0.67 & $0.27,1.66$ & 0.54 & $0.22,1.31$ & $0 \cdot 81$ & $0.64,1.03$ \\
\hline \multirow[t]{3}{*}{ Slovenia } & 1998 & $1 \cdot 36$ & $1 \cdot 20,1 \cdot 53$ & $1 \cdot 42$ & $1 \cdot 25,1 \cdot 60$ & $1 \cdot 29$ & $1 \cdot 15,1 \cdot 46$ & 1.09 & $0.96,1.23$ & $1 \cdot 01$ & $0.99,1.04$ \\
\hline & 2000 & $1 \cdot 52$ & $1 \cdot 35,1 \cdot 73$ & 1.54 & $1 \cdot 36,1 \cdot 74$ & 1.65 & $1 \cdot 46,1 \cdot 87$ & 1.23 & $1 \cdot 08,1 \cdot 39$ & $1 \cdot 05$ & $1.02,1.08$ \\
\hline & 2002 & $1 \cdot 43$ & $1 \cdot 26,1 \cdot 63$ & 1.58 & $1 \cdot 39,1 \cdot 80$ & $1 \cdot 70$ & $1.49,1.93$ & $1 \cdot 28$ & $1 \cdot 12,1 \cdot 45$ & $1 \cdot 07$ & $1 \cdot 04,1 \cdot 10$ \\
\hline Spa & 1991 & $1 \cdot 23$ & $1 \cdot 15,1 \cdot 31$ & 1.56 & $1.47,1.66$ & 1.65 & $1 \cdot 55,1 \cdot 75$ & 1.56 & $1 \cdot 46,1.66$ & $1 \cdot 13$ & $1 \cdot 11,1 \cdot 14$ \\
\hline Sweden & 1996 & $2 \cdot 36$ & $1 \cdot 71,3 \cdot 26$ & $2 \cdot 46$ & $1 \cdot 79,3 \cdot 39$ & $4 \cdot 11$ & $2 \cdot 87,5 \cdot 89$ & $5 \cdot 13$ & $3.47,1.63$ & $1 \cdot 49$ & $1.37,1.63$ \\
\hline
\end{tabular}

Source: The DAFNE databank (http://www.nut.uoa.gr/dafnesoft).

*The food expenditure ratio equals the household's food expenditures (incl. eating out of home) divided by the total household expenditures; increasing ratio reflects lower socio-economic status. Data adjusted for trimester of participation.

European countries during earlier HBS periods, households of higher socio-economic strata were less likely to be consumers of soft drinks and regularly purchased smaller quantities than their lower socio-economic counterparts. The exception in Central and Eastern European regions might indicate that in countries recently undergoing socio-economic transitions, soft drinks may have been considered as novel and trend-setting items.

Our observations are in line with previous studies indicating an increase in the consumption of soft drinks in recent years, also combined with an increase in the portion sizes offered ${ }^{(24,25)}$. In addition, a European study comparing the consumption of soft drinks among adults in ten countries using data collected through standardized $24 \mathrm{~h}$ dietary recalls also reported that soft drinks are a characteristic of the diet in North European and Scandinavian countries, but not in South Europe and France ${ }^{(13)}$. According to results of a different cross-national European study on the consumption of fruits and soft drinks among young adolescents, pupils in Scandinavian countries were significantly less likely to consume soft drinks daily than pupils in all other European areas ${ }^{(14)}$. According to the HBS data used in the present study and including all ages in each country's population, in the late 1990s daily availability was generally low in Finland and Sweden, but not in Norway.
In terms of food choices associated with soft drink consumption, our observation that soft drinks may displace milk in the daily diet has previously been reported through both observational and intervention studies ${ }^{(21,22,26)}$.

Our finding that households of lower social strata purchase more soft drinks agrees with that of a study analysing the influence of socio-economic status on the consumption of fruits and soft drinks among adolescents in a wide range of European countries ${ }^{(14)}$. Using various models to account for the adolescents' family characteristics, school environment and country of residence, the authors concluded that the daily consumption of soft drinks was significantly lower among pupils of parents of higher occupations for all areas except the Central and Eastern European countries. The authors further reported that in Central/Eastern European countries there was a significant increase in daily soft drink consumption with increasing family affluence, in line with our observation of higher availability of soft drinks among households of higher socio-economic status, and presumably higher prosperity, in Central/Eastern Europe, particularly in earlier years.

The data used in the present analysis were collected through the national HBS and cover all food items available for consumption to the household members for a 
Table 4 Percentage difference (\% Dif) and $95 \%$ confidence intervals in soft drink availability among consumers only. Results presented by quintile of household food expenditure ratio*, using the first quintile as referent

\begin{tabular}{|c|c|c|c|c|c|c|c|c|c|c|c|}
\hline \multirow[b]{2}{*}{ Country } & \multirow[b]{2}{*}{ Year of HBS } & \multicolumn{2}{|c|}{ 2nd quintile } & \multicolumn{2}{|c|}{ 3rd quintile } & \multicolumn{2}{|c|}{ 4th quintile } & \multicolumn{2}{|c|}{ 5th quintile } & \multicolumn{2}{|c|}{ Ordered quintiles } \\
\hline & & $\%$ Dif & $95 \% \mathrm{Cl}$ & $\%$ Dif & $95 \% \mathrm{Cl}$ & $\%$ Dif & $95 \% \mathrm{Cl}$ & $\%$ Dif & $95 \% \mathrm{Cl}$ & $\%$ Dif & $95 \% \mathrm{Cl}$ \\
\hline Austria & 1999 & +14 & $-2,+33$ & +4 & $-10,+19$ & +26 & $+10,+44$ & +39 & $+21,+60$ & +8 & $+5,+11$ \\
\hline \multirow[t]{2}{*}{ Cyprus } & 1997 & +15 & $+8,+22$ & +26 & $+19,+35$ & +28 & $+20,+37$ & +53 & $+42,+63$ & +10 & $+8,+11$ \\
\hline & 2003 & +12 & $+5,+19$ & +28 & $+20,+36$ & +42 & $+34,+51$ & +83 & $+71,+96$ & +15 & $+14,+17$ \\
\hline \multirow{3}{*}{ Finland } & 1985 & +3 & $-3,+9$ & +14 & $+7,+21$ & +22 & $+15,+30$ & +36 & $+27,+45$ & +8 & $+7,+10$ \\
\hline & 1990 & +10 & $+3,+17$ & +10 & $+3,+17$ & +22 & $+15,+30$ & +38 & $+29,+46$ & +8 & $+6,+9$ \\
\hline & 1998 & +9 & $0,+19$ & +10 & $+1,+20$ & +21 & $+11,+32$ & +44 & $+32,57$ & +9 & $+7,+11$ \\
\hline Germany & 1998 & -15 & $-22,-7$ & -11 & $-17,-3$ & -9 & $-16,-1$ & +4 & $-4,+12$ & +2 & $+1,+4$ \\
\hline \multirow[t]{2}{*}{ Greece } & 1998 & +4 & $-2,+9$ & +2 & $-3,+7$ & +5 & $+0,+11$ & +10 & $+4,+16$ & +2 & $+1,+3$ \\
\hline & 2004 & +9 & $+3,+15$ & +7 & $+1,+13$ & +7 & $+2,+13$ & +24 & $+18,+31$ & +4 & $+3,+6$ \\
\hline \multirow[t]{2}{*}{ Ireland } & 1994 & +5 & $+1,+9$ & +15 & $+11,+20$ & +24 & $+19,+28$ & +33 & $+28,+39$ & +8 & $+7,+9$ \\
\hline & 1999 & +20 & $+15,+25$ & +25 & $+21,+30$ & +42 & $+37,+48$ & +41 & $+34,+47$ & +9 & $+8,+10$ \\
\hline \multirow[t]{3}{*}{ Italy } & 1990 & +16 & $8,+24$ & +12 & $+4,+20$ & +16 & $+8,+24$ & +27 & $+19,+36$ & +5 & $+3,+6$ \\
\hline & 1993 & +8 & $+1,+15$ & +14 & $+7,+21$ & +24 & $+17,+32$ & +33 & $+25,+41$ & +7 & $+6,+9$ \\
\hline & 1996 & +8 & $+0,+17$ & +14 & $+5,+23$ & +22 & $+13,+33$ & +21 & $+12,+31$ & +5 & $+3,+7$ \\
\hline \multirow[t]{3}{*}{ Malta } & 1994 & -2 & $+1,+5$ & +4 & $+1,+12$ & +9 & $+1,+16$ & +17 & $+9,+26$ & +4 & $+3,+6$ \\
\hline & 1995 & +13 & $+6,+22$ & +16 & $+8,+25$ & +26 & $+18,+35$ & +27 & $+18,+35$ & +6 & $+4,+8$ \\
\hline & 2000 & +12 & $+4,+20$ & +20 & $+12,+28$ & +19 & $+10,+28$ & +46 & $+35,+57$ & +8 & $+7,+10$ \\
\hline Norway & 1997 & +14 & $-2,+33$ & +4 & $-10,19$ & +26 & $+10,+44$ & +39 & $+21,+60$ & +8 & $+5,+11$ \\
\hline \multirow[t]{3}{*}{ Portugal } & 1990 & -6 & $-13,+1$ & +1 & $-7,+9$ & -1 & $-8,+8$ & -18 & $-26,-11$ & -3 & $-5,-1$ \\
\hline & 1995 & +1 & $-6,+10$ & +12 & $+4,+22$ & +13 & $+4,+22$ & +15 & $+6,+25$ & +4 & $+2,+6$ \\
\hline & 2000 & -2 & $-8,+5$ & +1 & $-6,+8$ & +5 & $-3,+12$ & +11 & $+3,+20$ & +3 & $+1,+5$ \\
\hline \multirow[t]{3}{*}{ Slovak Republic } & 1997 & +7 & $-5,+21$ & +7 & $-7,+26$ & +39 & $+23,+58$ & +36 & $+20,+53$ & +9 & $+6,+12$ \\
\hline & 2000 & +1 & $-8,+11$ & -6 & $-15,+4$ & +7 & $-1,+17$ & +4 & $-5,+14$ & +1 & $-1,+4$ \\
\hline & 2003 & -4 & $-13,+4$ & +2 & $-6,+12$ & -10 & $-8,-1$ & +12 & $+3,+22$ & +2 & $0,+4$ \\
\hline \multirow[t]{3}{*}{ Slovenia } & 1998 & +6 & $-5,+17$ & +11 & $+0,+24$ & -2 & $-12,+9$ & +15 & $+4,+29$ & +2 & $0,+4$ \\
\hline & 2000 & +3 & $-8,+14$ & +4 & $-7,+16$ & +13 & $+1,+26$ & +27 & $+13,+43$ & $+\overline{6}$ & $+3,+9$ \\
\hline & 2002 & +9 & $-3,+22$ & +33 & $+19,+49$ & +46 & $+31,+63$ & +76 & $+56,+98$ & +15 & $+12,+18$ \\
\hline Spain & 1991 & +13 & $+5,+21$ & +8 & $+1,+16$ & +14 & $+7,+23$ & +21 & $+13,+29$ & +4 & $+2,+5$ \\
\hline Sweden & 1996 & -20 & $-33,-4$ & -3 & $-19,+15$ & +9 & $-8,+29$ & +18 & $-2,+42$ & +8 & $+3,+12$ \\
\hline
\end{tabular}

Source: The DAFNE databank (http://www.nut.uoa.gr/dafnesoft).

${ }^{*}$ The food expenditure ratio equals the household's food expenditures (incl. eating out of home) divided by the total household expenditures; increasing ratio reflects lower socio-economic status. Data adjusted for trimester of participation.

specified period of time. Among the strengths of the present study are the nationally representative population samples, the standardized data collection at regular time intervals, the subsequent harmonization of the available information to allow for inter-country comparisons, and the use of an indicator of socio-economic status which has been reported to adequately reflect social strata ${ }^{(18,20)}$. Socio-economic status can be assessed through various indicators. It is, however, generally acknowledged that no indicator is clearly superior to another and the use of different ones usually provides complementary insights ${ }^{(27)}$.

The study also has some weaknesses imposed by the nature of the data. The lack of information on eating out is an important limitation and is likely to affect estimations of soft drinks consumption ${ }^{(28)}$. A second limitation of the HBS data relates to different recording periods used in some of the countries. However, this inherent weakness will not affect within-country comparisons and no major bias is expected to be introduced when comparisons between countries are made. The HBS data refer to aggregate household acquisitions and a process of individualization is required. There are different ways to estimate the per person availability of foods and beverages; methods range from a simple division by the number of household members to the application of statistical modelling for calculating age- and genderspecific values ${ }^{(29,30)}$. In our analysis we have assumed an equal distribution of food among the household members and this is likely to affect the estimates, particularly in households where members of various age groups are present. The years of data collection are not strictly comparable among countries. Our working assumption, however, is that variability among countries is larger than that over time periods, at least for the outlying countries.

In the current paper we observed between- and withincountries disparities in the household consumption of soft drinks across Europe. High availability was generally reported among households of lower socio-economic status in Western and Northern Europe and it appears to be steadily and significantly increasing. Given the nature of our data we were not in a position to document which members of the household consumed these beverages, although children's diet often mirrors that of their parents $^{(10,31)}$. Soft drinks were also found to be correlated with unfavourable dietary choices such as higher availability of processed meat, sugar and sugar products and lower availability of plant foods and milk. These dietary patterns are likely to be shaped by advertising, the availability of vending machines in many schools and working places, and the relatively inadequate promotion 
of healthy food choices. Sources of comparable betweencountries information about long-term trends of food choices at home and their sociodemographic determinants can be essential in policy planning.

\section{Acknowledgements}

Sources of funding: The DAFNE initiative has been supported by the following EU projects: Cooperation in Science and Technology with Central and Eastern European Countries; the COST Action 99 - Food Consumption and Composition Data; the Agriculture and Agro-Industry, including fisheries (AIR) and the Agriculture and Fisheries (FAIR) Programmes; the Health Monitoring Programme of DG-SANCO; and the FP6-Specific Measures in Support of International Cooperation for Western Balkan Countries of DG-RESEARCH. Conflict of interest declaration: None to disclose. Authors' contributions: A.N. was the coordinator for the analyses in this paper and for drafting the manuscript. V.B. was the principal biostatistician in this study. A.T. is the Principal Investigator of the European DAFNE initiative continuously since 1994. The DAFNE participants contributed the HBS data of their own countries and collaborated in rendering the HBS data comparable between countries. Thanks are due to the Statistical Offices of all countries of the DAFNE network for supplying their national household budget survey data and supporting documentation, and for their unreserved collaboration.

\section{References}

1. Ludwig DS, Peterson KE \& Gortmaker SL (2001) Relation between consumption of sugar-sweetened drinks and childhood obesity: a prospective, observational analysis. Lancet 357, 505-508.

2. Malik VS, Schulze MB \& Hu FB (2006) Intake of sugarsweetened beverages and weight gain: a systematic review. Am J Clin Nutr 84, 274-288.

3. Palmer JR, Boggs DA, Krishnan $S$ et al. (2008) Sugarsweetened beverages and incidence of type 2 diabetes mellitus in African American women. Arch Intern Med 168, $1487-1492$.

4. Schulze MB, Manson JE, Ludwig DS et al. (2004) Sugarsweetened beverages, weight gain, and incidence of type 2 diabetes in young and middle-aged women. JAMA 292, 927-934.

5. Tucker KL, Morita K, Qiao N et al. (2006) Colas, but not other carbonated beverages, are associated with low bone mineral density in older women: The Framingham Osteoporosis Study. Am J Clin Nutr 84, 936-942.

6. Fung TT, Malik V, Rexrode KM et al. (2009) Sweetened beverage consumption and risk of coronary heart disease in women. Am J Clin Nutr 89, 1037-1042.

7. Bawa S (2005) The role of the consumption of beverages in the obesity epidemic. $J R$ Soc Health 125, 124-128.

8. Choi HK \& Curhan G (2008) Soft drinks, fructose consumption, and the risk of gout in men: prospective cohort study. BMJ 336, 285-286.

9. Branca F, Nikogocian $\mathrm{H} \&$ Lobstein T (editors) (2007) The Challenge of Obesity in the WHO European Region and The
Strategies for Response. Copenhagen: WHO Regional Office for Europe.

10. Bere E, Sørli Glomnes E, J te Velde S et al. (2007) Determinants of adolescents' soft drink consumption. Public Health Nutr 11, 49-56.

11. Gibson S \& Neate D (2007) Sugar intake, soft drink consumption and body weight among British children: further analysis of National Diet and Nutrition Survey data with adjustment for under-reporting and physical activity. Int J Food Sci Nutr 58, 445-460.

12. Libuda L, Alexy U, Buyken AE et al. (2008) Pattern of beverage consumption and long-term association with body-weight status in German adolescents - results from the DONALD study. Br J Nutr 99, 1370-1379.

13. Slimani N, Fahey M, Welch AA et al. (2002) Diversity of dietary patterns observed in the European Prospective Investigation into Cancer and Nutrition (EPIC) project. Public Health Nutr 5, 1311-1328.

14. Vereecken CA, Inchley J, Subramanian SV et al. (2005) The relative influence of individual and contextual socioeconomic status on consumption of fruit and soft drinks among adolescents in Europe. Eur J Public Health 15, 224-232.

15. Trichopoulou A \& Naska A (editors) (2001) The DAFNE initiative. Assessment of dietary patterns across Europe using household budget survey data. Public Health Nutr $\mathbf{4}$, 1129-1198.

16. European Communities (2003) Household Budget Surveys in the EU - Methodology and Recommendations for Harmonization. Luxembourg: Office for Official Publications of the European Communities.

17. Giskes K, Van Lenthe FJ, Brug J et al. (2007) Socioeconomic inequalities in food purchasing: the contribution of respondent-perceived and actual (objectively measured) price and availability of foods. Prev Med 45, 41-48.

18. James PT, Nelson M, Ralph A et al. (1997) Socio-economic determinants of health. The impact of nutrition to inequalities in health. BMJ 315, 1545-1549.

19. Engel E (1857) Die Productions- und Consumptionsverhältnisse des Königreichs Sachsen. Zeitschrift des Statistischen Büros des Königlich Sächsischen Ministeriums des Innern, 8/9. Reprinted as attachment to: Engel E (1895) Die Lebenskosten belgischer Arbeiter-Familien früher und jetzt. Int Stat Inst Bull 9, 1-74 (in German).

20. Trichopoulou A, Naska A, Costacou T \& the DAFNE III Group (2002) Disparities in food habits across Europe. Proc Nutr Soc 61, 553-558.

21. Keller KL, Kirzner J, Pietrobelli A et al. (2009) Increased sweetened beverage intake is associated with reduced milk and calcium intake in 3- to 7-year-old children at multi-item laboratory lunches. J Am Diet Assoc 109, 497-501.

22. Linardakis M, Sarri K, Pateraki MS et al. (2008) Sugar-added beverage consumption among kindergarten children of Crete: effects of nutritional status and risk of obesity. $B M C$ Public Health 8, 279.

23. Vartanian LR, Schwartz MB \& Brownell KD (2007) Effects of soft drink consumption on nutrition and health: a systematic review and meta-analysis. Am J Public Health 97, 667-675.

24. Nielsen SJ \& Popkin BM (2003) Patterns and trends in food portion sizes, 1977-1998. JAMA 289, 450-453.

25. Rugg-Gunn AJ, Fletcher ES, Matthews JN et al. (2007) Changes in consumption of sugars by English adolescents over 20 years. Public Health Nutr 10, 354-363.

26. Vågstrand K, Linné Y, Karlsson J et al. (2009) Correlates of soft drink and fruit juice consumption among Swedish adolescents. Br J Nutr 101, 1541-1548.

27. Kunst AE, Bos V, Mackenbach JP \& the EU Working Group on Socio-economic inequalities in Health (2001) Monitoring Socio-economic Inequalities in Health in the European 
Union: Guidelines and Illustrations. A Report for the Health Monitoring Program of the European Commission. Rotterdam: Erasmus University.

28. Orfanos P, Naska A, Trichopoulos D et al. (2007) Eating out of home and its correlates in 10 European countries. The European Prospective Investigation into Cancer and Nutrition (EPIC) study. Public Health Nutr 10, 1515-1525.

29. Chesher A (1997) Diet revealed? Semiparametric estimation of nutrient intake-age relationships. J R Stat Soc Ser A 160, 389-428.
30. Vasdekis VG, Stylianou S \& Naska A (2001) Estimation of age- and gender-specific food availability from household budget survey data. Public Health Nutr $\mathbf{4}$, 1149-1151.

31. Elfhag K, Tholin S \& Rasmussen F (2008) Consumption of fruit, vegetables, sweets and soft drinks are associated with psychological dimensions of eating behaviour in parents and their 12-year-old children. Public Health Nutr 11, 914-923.

\section{Appendix}

\section{DAFNE Participants}

Austria: I. Elmadfa and H. Freisling.

Belgium: A.M. Remaut-de-Winter and A.P. Cueto Eulert.

Croatia: K. Antonic Degac, M. Kamenski, D. Katic, M. Butigan, Z. Laido and A. Kaic-Rak.

Cyprus: E. Markidou, K. Onisiforou and A. Agrotou.

Finland: M.A. Berg, A. Pajunen and T. Hirvonen.

France: J.L. Volatier and J. Maffre.

Germany: G. Karg, K. Gedrich and K. Wagner.

Greece: A. Trichopoulou (project coordinator), A. Naska, V. Bountziouka, Y. Chloptsios, E. Oikonomou and K. Tsiotas. Hungary: G. Zajkas and P. Szivos.

Italy: A. Turrini, S. Barcherini and S. Martines.

Latvia: N. Petruhina, L. Sparite and D. Šantare.

Luxembourg: J. Langers, A. Schmitt and M. Zanardelli.

Malta: L. Pace, E. Caruana and N. Camilleri.

Montenegro: M. Burzanovic, Z. Savic, N. Terzic and L. Zizic.

Norway: K. Trygg, E. Mork and K. Lund-Iversen.

Poland: W. Sekula, A. Bienkuska, M. Morawska and Z. Niedzialek.

Portugal: M.D. Vaz de Almeida and S. Rodrigues.

Republic of Ireland: C. Kelleher and S. Friel.

Republic of Serbia: Z. Jovanovski and V. Božanić.

Slovak Republic: E. Leskova and H. Sukenikova.

Slovenia: M. Gabrijelcic, M. Adamic, M. Gregoric and M. Remec.

Spain: O. Moreiras, C. Cuadrado, M.L. Boned and P. Seoane Spiegelberg.

Sweden: M. Sjostrom, A. Yngve and E. Poortvliet.

United Kingdom: M. Nelson, D. Rimmer and S. Burr. 